\title{
O impacto do câncer de mama na saúde sexual feminina: uma revisão de literatura
}

\author{
The impact of breast cancer on female sexual health: a literature review
}

El impacto del cáncer de mama en la salud sexual femenina: una revisión de la literatura

Raysa Maria Silva de Araujo ${ }^{1}$, Danielle Laís Lopes Barboza ${ }^{2}$, Daniela Winckler Mass ${ }^{2}$, Igor dos Santos Cavalcante ${ }^{2 \star}$, Luciana Eda Maximiano Hasegawa ${ }^{1}$, Martha Laura Leão dos Santos Silva ${ }^{2}$, Ana Vitória Meireles Veiga ${ }^{2}$, Gabriel Phelipe Dantas do Nascimento'2, Thiago Santos Lima Almendra ${ }^{2}$, Lúcia Maria de Sousa Aguiar dos Santos ${ }^{3}$.

\section{RESUMO}

Objetivo: Descrever os efeitos que o câncer de mama exerce na saúde sexual feminina e de que forma ele impacta na qualidade de vida delas, abrangendo aspectos fisiológicos e psicológicos. Métodos: Revisão de Literatura Integrativa, cujos artigos foram buscados nas bases de dados SCIELO, MEDLINE E LILACS. Os critérios de inclusão foram artigos integrais publicados de 2000 a 2020, em português e inglês, e os critérios de exclusão foram artigos não relacionados ao tema. Resultados: A literatura demonstra relação clara e direta dos impactos negativos que o câncer de mama exerce na saúde feminina, evidenciando um quadro de libido reduzida, dispareunia, anorgasmia, ressecamento vaginal e ansiedade ou depressão. A realização da mastectomia representa fator de agravamento, afetando o bem-estar e saúde sexual feminina e causando sequelas na autoimagem corporal. Os resultados obtidos demonstram que a disfunção sexual é um dos principais efeitos negativos, comprometendo a saúde delas, especialmente no pós-cirúrgico. Considerações finais: Conclui-se que o câncer de mama pode prejudicar severamente a sexualidade das pacientes, gerando desfechos de acordo com o tratamento adotado. Por isso, é importante prevenir desfechos negativos e fornecer uma rede de apoio para as mulheres acometidas.

Palavras-chave: Neoplasias da mama, Sexualidade, Mastectomia.

\begin{abstract}
Objective: To describe the effects that breast cancer has on women's sexual health and the impact on women's quality of life, according to their physiological and psychological aspects. Methods: Integrative Literature Review, whose articles were searched in the SCIELO, MEDLINE AND LILACS databases. The inclusion criteria were full and free scientific articles published between 2000 and 2020, in Portuguese and English, and the exclusion criteria were articles unrelated to the topic. Results: The literature shows a clear and direct relationship of the negative impacts that breast cancer has on women's health, showing a reduced libido, dyspareunia, anorgasmia, vaginal dryness and anxiety or depression. Total mastectomy also represents another worsening factor, drastically affecting female sexual well-being and health, in addition to causing sequelae in body self-image. Thus, the results obtained demonstrate that dysfunction in women's sexuality is one of the main side effects in most cases, significantly compromising the health of patients, especially in the post-surgical period. Final considerations: It is concluded that breast cancer can severely affect patients' sexuality, generating negative outcomes according to the treatment adopted. For this reason, it is important to prevent the possible side effects of the disease and to provide a support network for women affected.
\end{abstract}

Keywords: Breast neoplasms, Sexuality, Mastectomy.

1 Instituto de Educação Superior do Vale do Parnaíba (IESVAP), Parnaíba - PI. *E-mail: igorsc@live.com

2 Universidade Federal do Delta do Parnaíba (UFDPar), Parnaíba - PI.

${ }^{3}$ Faculdade Integral Diferencial (FACID), Teresina - PI.

SUBMETIDO EM: 7/2020

ACEITO EM: 8/2020

PUBLICADO EM: 11/2020 


\section{RESUMEN}

Objetivo: Describir los efectos que el cáncer de seno tiene sobre la salud sexual de las mujeres y cómo afecta la vida de las mujeres, de acuerdo con sus aspectos fisiológicos y psicológicos. Métodos: Integrative Literature Review, cuyos artículos fueron buscados en las bases de datos SCIELO, MEDLINE Y LILACS. Los criterios de inclusión fueron artículos científicos completos y gratuitos publicados entre 2000 y 2020, en portugués e inglés, y los criterios de exclusión fueron artículos no relacionados con el tema. Resultados: La literatura demuestra una relación clara de los impactos negativos que el cáncer de seno tiene en la salud de las mujeres, mostrando una disminución de la libido, dispareunia, anorgasmia, sequedad vaginal y ansiedad o depresión. La mastectomía total también representa otro factor de empeoramiento, que afecta el bienestar y sexual femenina, además de causar secuelas en la autoimagen. Por lo tanto, los resultados obtenidos demuestran que la disfunción en la sexualidad de las mujeres es uno de los principales efectos secundarios en la mayoría de los casos, lo que compromete significativamente la salud de los pacientes. Consideraciones finales: Se concluye que el cáncer de seno puede afectar severamente la sexualidad de las pacientes, generando resultados negativos de acuerdo con el tratamiento. Por esta razón, es importante prevenir los efectos secundarios de la enfermedad y proporcionar una red de apoyo para las mujeres afectadas.

Palabras clave: Neoplasias de la mama, Sexualidad, Mastectomía.

\section{INTRODUÇÃO}

O câncer de mama é o tipo mais incidente de câncer em mulheres no mundo, correspondendo a $24,2 \%$ da totalidade dos casos de cânceres em 2018, além disso, representa a quinta causa de morte por câncer em geral e a mais recorrente no sexo feminino, com cerca de 2,1 milhões de casos novos por ano (WHO, 2018). No Brasil, com exceção dos tumores de pele não melanoma, essa neoplasia também é a mais ocorrente no sexo feminino e a estimativa para o ano de 2020 é de 66.280 casos novos (INCA, 2020).

A fisiologia envolvendo o desenvolvimento de um câncer de mama é bem elucidada no estudo desenvolvido por Duarte TP e Andrade AN (2003), no qual eles descrevem o processo como sendo causado pela multiplicação anormal das células em uma velocidade muito superior ao que fisiologicamente deveria acontecer. Esse desenvolvimento anormal pode se estender a estruturas vizinhas e causar comprometimento em diferentes sítios (metástases).

Os fatores de risco para a doença incluem a faixa etária (quatro em cada 5 casos acontecem depois dos 50 anos), a menarca precoce, a nuliparidade, a obesidade após a menopausa, o sedentarismo e o histórico familiar de câncer de ovário e de mama. Outro aspecto relevante é a alta taxa de mortalidade que se agrava por ser, frequentemente, uma patologia de diagnóstico tardio, o que aumenta a chance de insucesso no tratamento (BOMFIM IQM, et al., 2014).

Todavia, com uma detecção precoce e o desenvolvimento dos tratamentos oferecidos, observa-se que aproximadamente $50 \%$ das mulheres tratadas possuem uma sobrevida de, pelo menos, 15 anos após o diagnóstico e, desse modo, passam a ter que conviver com as consequências físicas e emocionais advindas da terapêutica utilizada (VERENHITACH BD, et al., 2014).

Após o diagnóstico, o tratamento pode ser realizado de maneira conservadora com a retirada de um segmento da mama a partir de uma quadrantectomia, ou de maneira não conservadora, isto é, com a retirada total da mama (mastectomia). Além disso, outros tratamentos podem ser associados, como a radioterapia, quimioterapia, terapia-alvo e/ou hormonioterapia, os quais, geralmente, podem acarretar efeitos colaterais como quadros de atrofia vulvovaginal, dispareunia, irritação e secura vaginal, inibição do desejo ou excitação, menopausa prematura, náuseas, vômitos, fadiga, alopecia, entre outros (MARTINS JOA, et al., 2020).

Com efeito, após o tratamento, por vezes agressivo, faz-se necessária uma avaliação da qualidade de vida da paciente que, além da sua condição de saúde, inclui fatores como a percepção da sua imagem corporal, seus sentimentos e suas relações sociais (HUGUET PR, et al., 2009). Os questionamentos pessoais atribuídos a fatores como as modificações estéticas e fisiológicas advindas das terapêuticas empreendidas 
podem contribuir para uma nova percepção muitas vezes negativa da identidade visual, provocando um declínio da autoestima, que pode influenciar negativamente na vida sexual da mulher.

A sexualidade engloba elementos culturais, biológicos, subjetivos e relacionais, contemplando práticas físicas e mentais com a finalidade de se obter prazer de maneira individual ou a partir de relações interpessoais (VIEIRA EM, et al., 2014).

Além disso, esse mesmo fator, no decorrer da vida, pode passar por diferentes modificações, principalmente quando sucedido por uma enfermidade árdua como o câncer de mama. Desse modo, a vivência do câncer e o seu manejo devem ser realizadas de maneira integrativa, compreendendo não apenas os fatores físicos, mas também os psicológicos, sociais e culturais para impedir ou atenuar as repercussões negativas oriundas da doença (SANTOS DB, et al., 2014).

O presente estudo tem como objetivo assimilar a influência do câncer de mama e de sua terapêutica na sexualidade por meio de uma revisão integrativa da literatura com o estudo e a compilação de produções científicas nacionais e internacionais.

Dessa forma, possibilita-se uma visão integral da saúde da mulher e facilita a disseminação dos conhecimentos obtidos para, através do aumento da conscientização a respeito da problemática, diminuir a consternação física e emocional consequente da enfermidade.

\section{MÉTODOS}

Trata-se de uma revisão de literatura integrativa sobre o impacto que o câncer de mama pode exercer na saúde sexual feminina dentro de seus aspectos fisiológicos e psicológicos. A busca de artigos foi feita nas seguintes bases de dados: Scientific Eletronic Library Online (SciELO), Medical Literature Analysis and Retrievel System Online (Medline) e Literatura Latino-Americana e do Caribe (LILACS), utilizando os descritores: "Neoplasias da mama", "Sexualidade" e "Mastectomia".

Ao utilizar os três descritores na base LILACS, não foi encontrado nenhum resultado, em seguida, a busca foi feita com os descritores associados em pares: com "mastectomia" e "sexualidade" foram encontrados quinze artigos, as outras duas duplas de pares não apresentaram nenhum resultado.

$\mathrm{Na}$ base SciELO, ao utilizar os três descritores em associação foram encontrados cinco artigos, ao usar o par "mastectomia" e "sexualidade" foram encontrados oito artigos, utilizando o par "mastectomia" e "neoplasias da mama" foram encontrados cento e vinte e dois artigos e o outro par não foi obtido resultado.

Na base MEDLINE, ao utilizar os três descritores foram encontrados quarenta e dois artigos, ao usar o par "sexualidade" e "neoplasias da mama" foram encontrados cento e sessenta e quatro artigos, utilizando o par "mastectomia" e "neoplasias da mama" foram encontrados quatorze mil duzentos e noventa e sete, ao usar o par "mastectomia" e "sexualidade" foram encontrados quarenta e cinco artigos.

Dos quatorze mil seiscentos e noventa e oito artigos encontrados na primeira busca, foram descartados quatorze mil quinhentos e seis artigos após a leitura do título. Assim, restaram cento e noventa e dois artigos para a leitura do resumo.

Destes, foram descartados, após a leitura dos resumos, cento e sessenta e quatro artigos por não estarem diretamente relacionados ao estudo. Desse total, foram descartados doze artigos devido a repetição. Por conseguinte, foram selecionados dezoito artigos, descritos no Quadro 1, para a leitura integral para a composição desse estudo.

Os critérios de inclusão foram: artigos científicos disponibilizados de forma gratuita e integral, que estivessem enquadrados como pesquisas em seres humanos com ano de publicação entre 2000 e 2020 . Os critérios de exclusão foram: materiais que não fossem artigos científicos e estudos que não tivessem de acordo com o tema selecionado. 
Figura 1 - Fluxograma detalhando cada etapa do processo de seleção de artigos para a revisão.

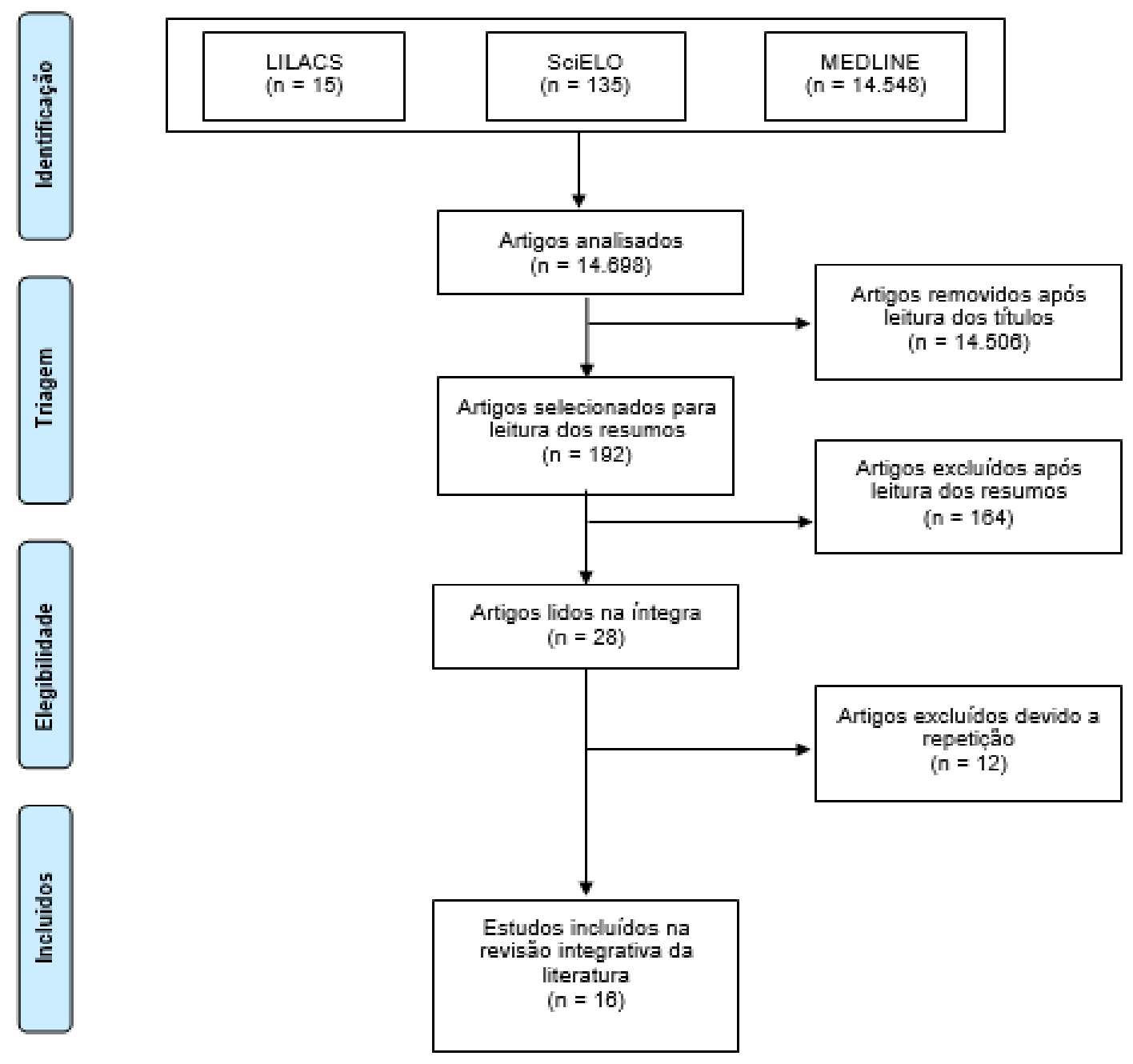

Fonte: ARAUJO RMS, et al., 2020.

\section{RESULTADOS E DISCUSSÃO}

Dos artigos encontrados a amostra final foi constituída por artigos indexados nas três bases de dados descritas e que contemplaram todos os critérios de inclusão utilizados para validar os estudos no processo de busca bibliográfica. Os artigos selecionados abrangeram revistas diferentes (Quadro 1).

No estudo desenvolvido por Martins JOA, et al. (2020), assim como no estudo produzido por Duarte TP e Andrade AN (2003), a presença do câncer de mama entre as mulheres brasileiras alcançou a primeira colocação nos rankings dos principais tipos de câncer que são registrados anualmente no Brasil. Como podemos observar, no intervalo de 17 anos, a realidade retratada pelos estudos publicados sobre o tema da mortalidade feminina envolvendo o câncer permaneceu praticamente a mesma. Mundialmente, o câncer de mama também é apontado como o principal tipo de câncer responsável pela morte de mulheres (DUARTE TP e ANDRADE AN, 2003).

Nos artigos analisados, percebe-se que o câncer de mama influencia a saúde da mulher em vários aspectos, mas, principalmente, no que tange à sexualidade e a percepção da imagem corporal (HUBER C, et al., 2006). Os resultados mais encontrados foram o de piora na qualidade do ato sexual em si, devido a alterações nos atributos que propiciam um ambiente de excitação entre os parceiros. Insegurança quanto à atratividade do corpo feminino, falta de lubrificação e diminuição da libido, foram, também, algumas características relatadas (HART AM, et al., 2015). 
Revista Eletrônica Acervo Saúde / Electronic Journal Collection Health | ISSN 2178-2091

Quadro 1 - Artigos selecionados para a composição do estudo, abordando a revista, ano de publicação, objetivos e seus respectivos resultados principais.

\begin{tabular}{|c|c|c|c|c|}
\hline Nome do Artigo & Revista & $\begin{array}{c}\text { Ano de } \\
\text { Publicação }\end{array}$ & Objetivos do Artigo & Principais Resultados \\
\hline $\begin{array}{l}\text { Enfrentando A Mastectomia: } \\
\text { Análise Dos Relatos De } \\
\text { Mulheres Mastectomizadas } \\
\text { Sobre Questões Ligadas À } \\
\text { Sexualidade }\end{array}$ & $\begin{array}{l}\text { Estudos De } \\
\text { Psicologia }\end{array}$ & 2003 & $\begin{array}{l}\text { Investigar como seis mulheres } \\
\text { mastectomizadas, com idades de } 37 \\
\text { a } 55 \text { anos, e com tempo de cirurgia } \\
\text { de } 1 \text { ano e meio a } 8 \text { anos, percebiam } \\
\text { a sua sexualidade. }\end{array}$ & $\begin{array}{l}\text { Após a mastectomia, as mulheres apresentaram dificuldades em } \\
\text { lidar com situações de exposição do corpo. Apesar dos temores, } \\
\text { algumas mulheres produziram diversos modos de expressarem } \\
\text { sua sexualidade a fim de potencializar suas relações. }\end{array}$ \\
\hline $\begin{array}{l}\text { Sexuality And Intimacy Issues } \\
\text { Facing Women With Beast } \\
\text { Cancer }\end{array}$ & $\begin{array}{l}\text { Journal Club - } \\
\text { Oncology Nursing } \\
\text { Forum }\end{array}$ & 2006 & $\begin{array}{l}\text { Explorar as experiências de } \\
\text { sexualidade e intimidade } \\
\text { enfrentadas por mulheres com } \\
\text { câncer de mama. }\end{array}$ & $\begin{array}{l}\text { Falta conhecimento da paciente sobre a mastectomia e a } \\
\text { menopausa induzida por quimioterapia em relação à sexualidade } \\
\text { ao longo da vida. Os profissionais de saúde devem instituir } \\
\text { educação necessária e abrir linhas de comunicação. }\end{array}$ \\
\hline $\begin{array}{c}\text { Qualidade De Vida E } \\
\text { Sexualidade De Mulheres } \\
\text { Tratadas De Câncer De Mama }\end{array}$ & $\begin{array}{l}\text { Rev Bras Ginecol } \\
\text { Obstet }\end{array}$ & 2009 & $\begin{array}{l}\text { Avaliar a qualidade de vida e } \\
\text { aspectos da sexualidade de } \\
\text { mulheres com câncer de mama } \\
\text { segundo o tipo de cirurgia e } \\
\text { características sociodemográficas. }\end{array}$ & $\begin{array}{l}\text { Idade, escolaridade, tipo de cirurgia e tempo desde a cirurgia não } \\
\text { influenciaram na qualidade de vida. Mulheres com relacionamento } \\
\text { marital estável e aquelas com maior nível socioeconômico tiveram } \\
\text { escores maiores de qualidade de vida. As submetidas à } \\
\text { quadrantectomia ou à mastectomia e reconstrução tiveram } \\
\text { melhores escores quanto à atratividade quando comparadas às } \\
\text { mastectomizadas sem reconstrução. }\end{array}$ \\
\hline $\begin{array}{c}\text { Sexual Function After Breast } \\
\text { Cancer }\end{array}$ & J Sex Med & 2011 & $\begin{array}{l}\text { Avaliar o impacto do primeiro } \\
\text { diagnóstico de câncer de mama e } \\
\text { seu tratamento, sintomas da } \\
\text { menopausa e imagem corporal na } \\
\text { função sexual. }\end{array}$ & $\begin{array}{l}\text { Das } 1.011 \text { mulheres, } 70 \% \text { tiveram disfunção sexual e } 77 \% \\
\text { sintomas vasomotores. Estas tinham duas vezes mais chances de } \\
\text { ter problemas de função sexual, o que se intensificava naquelas } \\
\text { que usavam inibidores de aromatase. Mulheres que usam } \\
\text { tamoxifeno não eram mais propensas a disfunção sexual. }\end{array}$ \\
\hline $\begin{array}{l}\text { Implicações Da Mastectomia Na } \\
\text { Sexualidade E Imagem Corporal } \\
\text { Da Mulher E Resposta Da } \\
\text { Enfermagem Perioperatória }\end{array}$ & $\begin{array}{l}\text { Revista De } \\
\text { Enfermagem } \\
\text { Referência }\end{array}$ & 2011 & $\begin{array}{l}\text { Integrar e analisar dados de estudos } \\
\text { realizados, contribuindo para uma } \\
\text { melhor compreensão da conjuntura } \\
\text { atual e promovendo a crescente } \\
\text { qualidade dos cuidados prestados. }\end{array}$ & $\begin{array}{l}\text { Os resultados dos estudos analisados demonstram que a } \\
\text { depreciação da imagem corporal está diretamente relacionada com } \\
\text { a vivência da sexualidade, sendo que quanto mais mutilante for a } \\
\text { cirurgia, maior será a depreciação da imagem corporal. }\end{array}$ \\
\hline $\begin{array}{l}\text { Mastectomia E Sexualidade: } \\
\text { Uma Revisão Integrativa }\end{array}$ & $\begin{array}{c}\text { Psicologia: } \\
\text { Reflexão E Crítica }\end{array}$ & 2012 & $\begin{array}{l}\text { Investigar o impacto do câncer de } \\
\text { mama e da mastectomia na } \\
\text { sexualidade da mulher. }\end{array}$ & $\begin{array}{l}\text { Os resultados evidenciaram que, após a retirada da mama, o } \\
\text { desempenho sexual é comprometido, com redução da frequência } \\
\text { de intercurso sexual nos primeiros estágios do tratamento e } \\
\text { diminuição da excitação, interesse e satisfação sexual. }\end{array}$ \\
\hline
\end{tabular}

$\overline{\text { REAS/EJCH | Vol.12(11) | e4726 | DOI: https://doi.org/10.25248/reas.e4726.2020 Página } \mathbf{5} \text { de } \mathbf{1 1}}$ 
Revista Eletrônica Acervo Saúde / Electronic Journal Collection Health | ISSN 2178-2091

\begin{tabular}{|c|c|c|c|c|}
\hline Nome do Artigo & Revista & $\begin{array}{c}\text { Ano de } \\
\text { Publicação }\end{array}$ & Objetivos do Artigo & Principais Resultados \\
\hline $\begin{array}{l}\text { Desconfortos Físicos } \\
\text { Decorrentes Dos Tratamentos } \\
\text { Do Câncer De Mama } \\
\text { Influenciam A Sexualidade Da } \\
\text { Mulher Mastectomizada? }\end{array}$ & $\begin{array}{c}\text { Rev Esc Enferm } \\
\text { Usp }\end{array}$ & 2012 & $\begin{array}{l}\text { Analisar a produção científica sobre } \\
\text { sexualidade da mulher com câncer } \\
\text { de mama após a mastectomia, com } \\
\text { foco nos desconfortos físicos } \\
\text { decorrentes dos tratamentos. }\end{array}$ & $\begin{array}{l}\text { Os achados evidenciaram que, mesmo quando existe intensa e } \\
\text { satisfatória vida sexual no período prévio à doença, fatores como } \\
\text { estresse, dor, fadiga, insulto à imagem corporal e baixa autoestima, } \\
\text { decorrentes dos tratamentos, podem desorganizar } \\
\text { funcionamento sexual da mulher acometida. }\end{array}$ \\
\hline $\begin{array}{c}\text { Changes To Sexual Well-being } \\
\text { And Intimacy After Breast } \\
\text { Cancer }\end{array}$ & Cancer Nursing & 2012 & $\begin{array}{l}\text { Examinar a experiência vivida de } \\
\text { bem-estar sexual e intimidade do } \\
\text { casal em uma grande amostra de } \\
\text { indivíduos com câncer de mama } \\
\text { residentes na Austrália, usando uma } \\
\text { combinação de métodos } \\
\text { quantitativos e qualitativos. }\end{array}$ & $\begin{array}{l}\text { Diminuições na frequência sexual, resposta e satisfação foram } \\
\text { atribuídas a fatores como cansaço e dor, sofrimento psicológico e } \\
\text { imagem corporal, e alterações da menopausa induzidas por } \\
\text { medicamentos, como secura vaginal e ganho de peso. As } \\
\text { preocupações predominantes foram consequências emocionais, } \\
\text { mudanças físicas, sentimento não atraente ou falta de feminilidade, } \\
\text { reconciliação do eu com as mudanças e impacto no parceiro ou } \\
\text { relacionamento }\end{array}$ \\
\hline $\begin{array}{l}\text { The Sexual Life Of Women With } \\
\text { Breast Cancer: Meanings } \\
\text { Attributed To The Diagnosis And } \\
\text { Its Impact On Sexuality }\end{array}$ & $\begin{array}{l}\text { Estudos De } \\
\text { Psicologia } \\
\text { Campinas }\end{array}$ & 2013 & $\begin{array}{l}\text { Investigar a vida sexual da mulher } \\
\text { com câncer de mama no primeiro } \\
\text { ano após o procedimento cirúrgico, } \\
\text { buscando-se demarcar os } \\
\text { significados atribuídos ao } \\
\text { diagnóstico e suas repercussões na } \\
\text { sexualidade. }\end{array}$ & $\begin{array}{l}\text { Da análise temática emergiram duas categorias, evidenciando o } \\
\text { impacto tanto negativo como positivo do câncer na vida sexual. } \\
\text { Essa variedade de significados encontrados mostra que não existe } \\
\text { um padrão único de vivência sexual após o câncer. A maneira } \\
\text { como cada mulher ressignifica o adoecimento contribui para que } \\
\text { vivencie singularmente sua sexualidade. }\end{array}$ \\
\hline $\begin{array}{l}\text { Sexuality After Breast } \\
\text { Reconstruction Post } \\
\text { Mastectomy }\end{array}$ & Aesth Plast Surg & 2013 & $\begin{array}{l}\text { Avaliar e comparar a sexualidade } \\
\text { em mulheres submetidas a } \\
\text { mastectomia isoladamente com } \\
\text { aquelas submetidas a reconstrução } \\
\text { mamária após mastectomia. }\end{array}$ & $\begin{array}{l}\text { Os dados mostraram um escore FSFI menor para o grupo de } \\
\text { mastectomia isolada em comparação ao grupo de reconstrução } \\
\text { mamária. Não foi estabelecida relação entre os escores e o tempo } \\
\text { pós-operatório ou entre os escores e a receita de remuneração, } \\
\text { relacionamento estável e idade para ambos os grupos. A média de } \\
\text { idade não apresentou diferença significativa. }\end{array}$ \\
\hline $\begin{array}{c}\text { Câncer De Mama E Seus } \\
\text { Efeitos Sobre A Sexualidade: } \\
\text { Uma Revisão Sistemática Sobre } \\
\text { Abordagem E Tratamento }\end{array}$ & Femina & 2014 & $\begin{array}{l}\text { Revisar a literatura médica referente } \\
\text { aos efeitos do câncer de mama e } \\
\text { também o seu tratamento sobre a } \\
\text { sexualidade, considerando as } \\
\text { esferas psicossociais e biológicas, } \\
\text { além das abordagens semiológicas } \\
\text { e terapêuticas disponíveis. }\end{array}$ & $\begin{array}{l}\text { Estudos mostram alterações físicas decorrentes da quimioterapia, } \\
\text { hormonioterapia e tratamento cirúrgico que interferem na } \\
\text { sexualidade, promovendo disfunção no desejo, excitação, } \\
\text { lubrificação e orgasmo. Experiências psíquicas incluem medo da } \\
\text { perda da fertilidade, imagem corporal negativa, sentimento de não } \\
\text { ser sexualmente atraente, depressão e ansiedade. A qualidade } \\
\text { prévia do relacionamento com o parceiro é considerada o mais } \\
\text { importante fator preditivo da qualidade do relacionamento sexual } \\
\text { após o término do tratamento. }\end{array}$ \\
\hline
\end{tabular}

$\overline{\text { REAS/EJCH | Vol.12(11) | e4726 | DOI: https://doi.org/10.25248/reas.e4726.2020 Página } 6 \text { de } 11}$ 
Revista Eletrônica Acervo Saúde / Electronic Journal Collection Health | ISSN 2178-2091

\begin{tabular}{|c|c|c|c|c|}
\hline Nome do Artigo & Revista & $\begin{array}{c}\text { Ano de } \\
\text { Publicação }\end{array}$ & Objetivos do Artigo & Principais Resultados \\
\hline $\begin{array}{l}\text { Female Sexuality After Female } \\
\text { Cancer Treatment: A Clinical } \\
\text { Issue }\end{array}$ & $\begin{array}{c}\text { Eur J Gynaecol } \\
\text { Oncol }\end{array}$ & 2014 & $\begin{array}{l}\text { Registrar como o tratamento do } \\
\text { câncer feminino pode afetar a } \\
\text { sexualidade e as relações } \\
\text { interpessoais no casal. }\end{array}$ & $\begin{array}{l}\text { Uma redução significativa do desejo sexual, excitação sexual e } \\
\text { orgasmo foi encontrada em contraste com o grupo de controle. A } \\
\text { pontuação na qualidade do relacionamento aumentou } \\
\text { significativamente nos grupos de câncer. }\end{array}$ \\
\hline $\begin{array}{l}\text { Vivência Da Sexualidade Após } \\
\text { O Câncer De Mama: Estudo } \\
\text { Qualitativo Com Mulheres Em } \\
\text { Reabilitação }\end{array}$ & $\begin{array}{l}\text { Rev. Latino-am. } \\
\text { Enfermagem }\end{array}$ & 2014 & $\begin{array}{l}\text { Compreender as repercussões } \\
\text { psicossociais e culturais do câncer } \\
\text { de mama e seus } \\
\text { tratamentos na sexualidade de } \\
\text { mulheres. }\end{array}$ & $\begin{array}{l}\text { Relatos expressaram sentimentos de angústia e menos valia, } \\
\text { suscitados pelas alterações corporais após o tratamento, por } \\
\text { envolverem características físicas relacionadas aos modelos de } \\
\text { beleza e feminilidade culturalmente valorizados. As } \\
\text { recomendações fornecidas pelos profissionais de saúde foram, em } \\
\text { sua maioria, prescritivas, sem abrir espaço para o diálogo. }\end{array}$ \\
\hline $\begin{array}{l}\text { Avaliação Da Função Sexual } \\
\text { Em Um Grupo De } \\
\text { Mastectomizadas }\end{array}$ & $\begin{array}{l}\text { Revista Brasileira } \\
\text { de Promoção à } \\
\text { Saúde, }\end{array}$ & 2014 & $\begin{array}{l}\text { Avaliar a função sexual de mulheres } \\
\text { mastectomizadas. }\end{array}$ & $\begin{array}{l}\text { Encontrou-se um desempenho sexual de nulo a ruim em } 35,3 \% \\
\text { das mulheres, e só } 11,8 \% \text { apresentaram um escore favorável. } \\
\text { Quanto ao estado civil e escolaridade, } 47,1 \% \text { eram casadas e } \\
55,9 \% \text { apresentaram escolaridade até ensino fundamental }\end{array}$ \\
\hline $\begin{array}{l}\text { Sexualidade E Câncer De } \\
\text { Mama: Uma Revisão } \\
\text { Sistemática Da Literatura }\end{array}$ & $\begin{array}{l}\text { Saúde Soc. São } \\
\text { Paulo }\end{array}$ & 2014 & $\begin{array}{l}\text { Compreender como o câncer de } \\
\text { mama e seus tratamentos afetam a } \\
\text { vivência da sexualidade da mulher } \\
\text { acometida. }\end{array}$ & $\begin{array}{l}\text { Além de afetar a percepção de sua sexualidade, o resultado da } \\
\text { cirurgia pode ser percebido pela mulher como uma mutilação os } \\
\text { demais tratamentos para o câncer de mama podem acarretar } \\
\text { menopausa induzida. O profissional de saúde exerce importante } \\
\text { função nesse contexto. }\end{array}$ \\
\hline $\begin{array}{l}\text { The Psychosexual Impact Of } \\
\text { Partial And Total } \\
\text { Breast Reconstruction }\end{array}$ & $\begin{array}{c}\text { Annals Of Plastic } \\
\text { Surgery }\end{array}$ & 2015 & $\begin{array}{l}\text { Investigar a satisfação e as } \\
\text { expectativas dos pacientes por } \\
\text { cirurgia nas áreas sexual e imagem } \\
\text { corporal e avaliar os resultados } \\
\text { referentes ao bem-estar sexual após } \\
\text { a reconstrução da mama. }\end{array}$ & $\begin{array}{l}\text { Ambos os grupos foram submetidos à mastectomia, no que fez } \\
\text { reconstrução mamária a satisfação com a vida sexual e a imagem } \\
\text { corporal não se alterou. No grupo que fez mamoplastia redutora, } \\
\text { as pacientes relataram um aumento na percepção de seus } \\
\text { parceiros sobre elas como mulheres e ganhos na capacidade de } \\
\text { usar roupas provocantes. }\end{array}$ \\
\hline $\begin{array}{l}\text { Sexualidade De Mulheres } \\
\text { Submetidas À Mastectomia: } \\
\text { Identificação das Fases } \\
\text { Afetadas No Ciclo Da Resposta } \\
\text { Sexual }\end{array}$ & $\begin{array}{l}\text { Revista Online De } \\
\text { Pesquisa Cuidado } \\
\text { É Fundamental }\end{array}$ & 2020 & $\begin{array}{l}\text { Analisar a sexualidade das } \\
\text { mulheres mastectomizadas, } \\
\text { identificando quais as fases do ciclo } \\
\text { da resposta sexual são afetadas e } \\
\text { quais as disfunções desenvolvidas. }\end{array}$ & $\begin{array}{l}\text { O desempenho sexual da população estudada apresentou-se } \\
\text { heterogêneo, } 50 \% \text { apresentou padrão sexual satisfatório e } 50 \% \\
\text { algum tipo de comprometimento na sexualidade, e dentro deste } \\
\text { grupo, incluem-se as casadas, com menos de } 49 \text { anos e com grau } \\
\text { de instrução de nível fundamental. }\end{array}$ \\
\hline
\end{tabular}

Fonte: ARAUJO RMS, et al., 2020.

$\overline{\text { REAS/EJCH | Vol.12(11) | e4726 | DOI: https://doi.org/10.25248/reas.e4726.2020 Página } 7 \text { de } 11}$ 


\section{Percepção da imagem corporal}

A compreensão de que a sexualidade é um dos principais aspectos afetados pelo tratamento apresenta uma relevância muito significativa para a compreensão holística da saúde da mulher. No pós-cirúrgico, quando os efeitos sobre a saúde podem repercutir negativamente sobre a aceitação da situação pela qual a paciente se encontra, como apontado por Cesnik VM e Santos MA (2012), é que nota-se queda da autoestima da mulher.

No estudo produzido por Moniz PAF, et al. (2011), a questão do não reconhecimento do corpo pela mulher após a cirurgia ou da sensação de corpo incompleto que pode persistir ao longo do período de recuperação, gera uma visão depreciada da imagem corporal pela mulher e que pode gerar efeitos negativos na esfera psicossocial ao longo dos anos.

Ussher JM, et al. (2012) identificaram que as questões mais frequentemente relatadas foram relacionadas a sentimentos em torno da aparência e às percepções de outras pessoas como: "preocupações com imagem/atratividade corporal", observadas por $77 \%$ das mulheres, seguidas por "falta de confiança" (66,5\%); "não se sente desejável" (65\%) e "medo de rejeição" (46,5\%).

\section{Sexualidade e alterações fisiológicas}

Como levantado pelo estudo realizado por Martins JOA, et al. (2020), um aspecto muito válido a ser considerado sobre a saúde da mulher após um tratamento de câncer de mama é a questão da sua saúde sexual, pois, como mencionado no estudo realizado por Duarte TP e Andrade AN (2003) e no estudo de Moniz PAF, et al. (2011), as mamas fazem parte da estética sexual feminina como um conceito culturalmente formado e, por isso, o diagnóstico de doenças como um câncer mamário costuma gerar um enorme medo para as pacientes acometidas.

Vários são os elementos fisiológicos, sociais e psicológicos que proporcionam bem estar e satisfação no cotidiano de uma pessoa e na sua vida conjugal, e fatores como sexualidade e intimidade são importantes para a qualidade de vida. O relacionamento sexual de um casal, cuja mulher passou por um tratamento de câncer de mama, pode ser afetado negativamente devido a outros aspectos resultantes do processo como depressão, cansaço, ressecamento vaginal, diminuição da libido, que acabam por diminuir a funcionalidade sexual (HUBER C, et al., 2006).

Segundo Ussher JM, et al. (2012) a maioria das 1956 mulheres entrevistadas relatou uma diminuição na frequência do sexo (78\%), na energia para o sexo (76\%), na excitação sexual $(74 \%)$, em sentir-se desejável (73\%), no interesse em sexo (71\%), no prazer sexual (64\%), na satisfação com sexo (62\%) e na intimidade com o parceiro (60\%), corroborando com o achado da pesquisa de BOMFIM IQM, et al. (2014) na qual 88,2\% das mulheres tinham ao menos uma disfunção sexual, sendo que das 34 mulheres, $88,2 \%$ apresentaram dificuldade na excitação, $82,4 \%$ desejo sexual hipoativo, e 79,4\% referiram dispareunia e anorgasmia.

Um ponto muito importante, visto no estudo desenvolvido por Cesnik VM e Santos MA (2012 B), é com relação ao desenvolvimento do ressecamento vaginal que se observa principalmente em mulheres que precisam passar pela quimioterapia como parte do tratamento contra o câncer. Essa secura vaginal relatada pelas pacientes pode apresentar uma ligação importante com a queixa de dor e desconforto durante 0 ato sexual, a dispareunia. Na pesquisa feita por Ussher JM, et al. (2012) realizada com 1965 mulheres, demonstrou o cansaço $(71,0 \%)$ a secura vaginal $(63,3 \%)$ e depressão/ansiedade $(37,8 \%)$ como uns dos aspectos mais prevalentes no tratamento contra o câncer que afetou o bem-estar sexual.

\section{Tratamentos e bem estar}

Segundo Neto MS, et al. (2013), a mastectomia, por si só, pode gerar fortes alterações no bem-estar sexual de uma mulher). Em seu estudo, foram analisadas 36 pacientes oncológicas entre 18 e 60 anos, do Departamento de Plástica Cirúrgica da Universidade Federal de São Paulo, divididas em dois grupos. No grupo 1, estavam 17 mulheres que realizaram mastectomia somente; já no grupo 2 se encontravam mulheres que realizaram reconstrução completa da mama após sofrer a mastectomia. Para tal análise utilizou-se uma escala da função sexual constando o nível de desejo, excitação, lubrificação, orgasmo, satisfação e 
dispareunia. Ao final verificou-se que as mulheres que realizaram somente a mastectomia obtiveram pontuações menores na escala - aproximadamente metade da pontuação do grupo 2. Evidenciando, assim, a menor satisfação com a imagem corporal e a sexualidade para aquelas que ainda estão com as marcas da mastectomia (NETO MS, et al., 2013)

As consequências de um tratamento de câncer de mama, não somente pela mastectomia, geram impactos nos aspectos sociais, subjetivos e no comportamento sexual da mulher. PANJARI M, et al. (2011), ao analisar o impacto do diagnóstico do câncer de mama invasivo e do seu tratamento, juntamente com os sintomas de menopausa, e a imagem corporal na função sexual feminina constataram que, aproximadamente, $82 \%$ das entrevistadas relataram ter uma boa e satisfatória função sexual anteriormente ao diagnóstico; porém, ao retornarem após 12 semanas e responderem o mesmo questionário, verificou-se que $70 \%$ das mulheres vivenciavam problemas sexuais, devido aos sinais de menopausa, sintomas vasomotores, coincidindo com o início do uso de inibidores da aromatase, como tratamento adjuvante (LUCARELLI AP, et al., 2013).

Quanto aos resultados obtidos por BOMFIM IQM, et al. (2014) relativos ao desempenho e à satisfação sexual, a maioria das mulheres $(35,3 \%)$ analisadas na pesquisa foi classificada na categoria de nulo a ruim, 23,5 como ruim a desfavorável, $29,4 \%$ como desfavorável a regular e poucas atingiram o escore classificado de bom a excelente e regular a bom, com 5,9\% em ambos.

\section{Relação com o parceiro}

Ademais, em muitas falas das pacientes estudadas, a falta do apoio e compreensão dos seus parceiros/cuidadores eram apontados como sendo fatores que tendem a influenciar bastante sobre a qualidade da vida sexual no pós-tratamento ao câncer de mama.

A percepção que a mulher tem sobre o apoio recebido pelo parceiro é considerada elemento importante que favorece o enfrentamento da doença e a adaptação da vida sexual após os tratamentos, mesmo quando há alterações físicas da função sexual (SANTOS DB, et al., 2014).

Em seu estudo, Ussher JM, et al. (2012) analisou respostas de mulheres com câncer de mama perguntando se o parceiro sofreu alguma consequência negativa por causa do câncer de mama, os relatos mais comuns foram o medo de me machucar durante o sexo (52\%), falta de interesse em sexo (37\%), dificuldades na comunicação (34\%), cansaço (28\%) e mudança de papel (me ver como paciente) $(20 \%)$.

Porém, em alguns casos, o comportamento sexual pode não ser afetado, permanecendo como estava anterior ao tratamento, ou até melhorado, em razão do apoio emocional dado pelo companheiro (VIEIRA EM, et al., 2014). Cesnik VM, et al. (2013) detectaram, em seu estudo, impactos positivos da doença sobre a vida sexual das mulheres, como: ressignificação positiva de sua vida, a valorização da vida como fator facilitador para a cuidando da aparência física e a possibilidade de morte como fator de promoção à valorização da vida.

\section{Fatores socioeconômicos}

Diferentes fatores podem ser relacionados com o desenvolvimento de uma disfunção sexual em um paciente que passou por um tratamento tido como mais agressivo, como no caso de mulheres com câncer de mama. O que se observa na literatura utilizada para a composição desse estudo é que o nível socioeconômico e o grau de instrução, segundo Huguet PR, et al. (2009) e a idade e estado civil, segundo BOMFIM IQM, et al. (2014) foram fatores considerados importantes para caracterizar o perfil das mulheres em estudo e assim compreender a forma pela qual sua sexualidade era afetada com o emprego do tratamento escolhido.

O que se observou com o estudo de Martins JOA, et al. (2020), foi que o grau de instrução educacional é um fator bem importante para avaliação sexual das mulheres. Huguet PR, et al. (2009) observaram que mulheres com taxas mais altas de escolaridade tiveram melhor autopercepção da sexualidade. GANZ PA, et al. (2003) em seu estudo com mulheres norte-americanas, identificou que aquelas com maior escolaridade também tiveram melhores taxas de qualidade de vida. O que os estudos conseguiram perceber foi que, quanto menor o nível de escolaridade pior era o padrão sexual dessa mulher, ou seja, maior a prevalência de queixas com relação à sexualidade. 


\section{Abordagem profissional}

Um ponto muito importante mencionado no estudo de Martins JOA, et al. (2020), e no estudo de Cesnik VM e Santos MA (2012) é com relação ao papel do profissional da saúde no fornecimento de um atendimento capaz de contemplar a vertente sexual que aflige as pacientes. O que se observa é que durante os atendimentos pós-cirúrgicos, a relação entre as pacientes e os profissionais não supera a barreira do pudor e constrangimento das pacientes para abordar o assunto envolvendo a sexualidade. Assim, o profissional de saúde precisa se despir do avental de seus estereótipos para contemplar as necessidades individuais da mulher no âmbito do cuidado (SANTOS DB, et al., 2014)

Quando analisamos conjuntamente a abordagem não efetiva dos profissionais da saúde e a precária rede de apoio fornecida por alguns companheiros/cuidadores das pacientes ao longo do tratamento, inferimos que esses fatores podem ter efeitos negativos na recuperação da saúde da mulher, principalmente sobre a saúde mental da mesma. Segundo Santos DB, et al. (2014) a participação da mulher no processo de tomada de decisão em relação ao tipo de cirurgia pode contribuir para que ela obtenha melhor ajustamento psicológico e, consequentemente, menor impacto negativo na sexualidade.

Os diferentes estudos utilizados para compor o presente trabalho, valendo-se destacar o realizado por Verenhitach BD, et al. (2014), chama atenção para o número reduzido de estudos sobre a disfunção sexual feminina na literatura. Lembrando que esse tema apresenta uma grande importância para o desenvolvimento de novas técnicas e para o fornecimento de um atendimento capaz de contemplar diferentes aspectos da saúde feminina e na conduta clínica dos diferentes profissionais relacionados a área oncológica. Constatouse a necessidade de desenvolver estudos sobre a formação do profissional de saúde voltada à sexualidade e pesquisas sobre a intervenção na atenção à sexualidade da mulher acometida (SANTOS DB, et al., 2014).

\section{CONSIDERAÇÕES FINAIS}

Entre todas as neoplasias presentes no Brasil, o câncer de mama teve maior incidência na população feminina. Diante do exposto na literatura, é possível concluir que a sexualidade feminina, fator essencial na vida da mulher, é fortemente influenciada pela abordagem terapêutica escolhida para o tratamento do câncer. Logo, evidencia-se a importância do diálogo com a paciente e a escolha junto ao médico do melhor tratamento, almejando-se prevenir precocemente os possíveis efeitos colaterais. A percepção do próprio corpo e outros quesitos que envolvem a sexualidade, como o desejo sexual, acabam por sofrer um impacto significativo em boa parte das pacientes. Faz-se necessário, então, o entendimento dos profissionais de saúde envolvidos na linha de cuidado a mulheres diagnosticadas com câncer de mama, trabalhando de forma multiprofissional e interdisciplinar, para minimizar as alterações decorrentes do tratamento. Esta premissa traz a importância da orientação da mulher e das pessoas que a cercam, como uma rede de apoio, a fim de minimizar os efeitos psicológicos da doença.

\section{REFERÊNCIAS}

1. BOMFIM IQM, et al. Avaliação da função sexual em um grupo de mastectomizadas. Rev Bras Promoç Saúde, Fortaleza, 2014; 27(1): 77-84.

2. CESNIK VM, et al. The sexual life of women with breast cancer: meanings attributed to the diagnosis and its impact on sexuality. Estudos de Psicologia, 2013; 30(2): 187-197.

3. CESNIK VM, SANTOS MA. Desconfortos físicos decorrentes dos tratamentos do câncer de mama influenciam a sexualidade da mulher mastectomizada? Rev. esc. enferm. USP, 2012; 46(4): 1001-1008.

4. CESNIK VM, SANTOS MA. Mastectomia e sexualidade: uma revisão integrativa. Psicologia: Reflexão e Crítica, 2012; 25(2): 339-349.

5. DUARTE TP, ANDRADE AN. Enfrentando a mastectomia: análise dos relatos de mulheres mastectomizadas sobre questões ligadas à sexualidade. Estudos de Psicologia, 2013; 8(1): 155-163.

6. ELISABETH MV, et al. Vivência da sexualidade após o câncer de mama: estudo qualitativo com mulheres em reabilitação. Rev. Latino-Am. Enfermagem, 2014; 22(3): 408-14.

7. GANZ PA, et al. Cancer in younger women: reproductive and late health effects of treatment. J Clin Oncol. 2003;21(22):4184-93.

8. HART AM, et al. The Psychosexual Impact of Partial and Total Breast Reconstruction. Annals of Plastic Surgery, 2015; $75(3): 281-6$. 
9. HUBER C, et al. Sexuality and Intimacy Issues Facing Women With Breast Cancer. Oncology Nursing Forum, 2006; 1163-7.

10. HUGUET PR, et al. Qualidade de vida e sexualidade de mulheres tratadas de câncer de mama. Rev. Bras. Ginecol. Obstet, 2009; 31(2): 61-67.

11. MARTINS JOA, et al. Sexualidade de mulheres submetidas à mastectomia: identificação das fases afetadas no ciclo da resposta sexual. Rev Fun Care Online, 2020; 12: 67-72.

12. INSTITUTO NACIONAL DE CÂNCER (BRASIL). Tipos de câncer: câncer de mama, 2020.

13. MONIZ PAF, et al. Implicações da mastectomia na sexualidade e imagem corporal da mulher e resposta da enfermagem perioperatória. Rev. Enf. Ref, 2011; (5): 163-171.

14. NETO MS, et al. Sexuality After Breast Reconstruction Post Mastectomy. Aesth Plast Surg, $2013 ; 37643-64$.

15. PANJARI M, et al. Sexual function after breast cancer. J Sex Med, 2011; 8:294-302.

16. SANTOS DB, et al. Sexualidade e câncer de mama: uma revisão sistemática da literatura. Saúde soc, 2014; 23(4): $1342-1355$.

17. USSHER JM, et al. Changes to Sexual Well-Being and Intimacy After Breast Cancer. Cancer Nursing, 2012; 35(6):456465.

18. VAIDAKIS D, et al. Female sexuality after female cancer treatment: a clinical issue. European Journal of Gynaecological Oncology, 2014; 35(6): 635-640.

19. VERENHITACH BD, et al. Câncer de mama e seus efeitos sobre a sexualidade: uma revisão sistemática sobre abordagem e tratamento. Femina, 2014; 42(1): 3-10.

20. WORLD HEALTH ORGANIZATION. International Agency for Research on Cancer: breast cancer, 2018. 